\title{
The association between the IFIH1 locus and type 1 diabetes
}

\author{
H.-Q. Qu • L. Marchand • R. Grabs • C. Polychronakos
}

Received: 18 September 2007 / Accepted: 9 November 2007 / Published online: 11 December 2007

(C) Springer-Verlag 2007

\begin{abstract}
Aims/hypothesis We set out to validate a recently reported type 1 diabetes association from the $I F I H I$ gene variation in an independent cohort from a population of mixed European descent.

Methods We genotyped five single-nucleotide polymorphisms in the IFIH1 locus, i.e. rs2111485, rs1990760, rs3747517, rs17783344 and rs984971589, in 589 type 1 diabetes nuclear family trios (1,767 individuals).

Results This study independently replicated the reported genetic association using a family-based approach.

Conclusions/interpretation The reported type 1 diabetes association is from a linkage disequilibrium region including three candidate genes, i.e. FAP, IFIHI and GCA. Further variant discovery and fine mapping could help clarify a novel type 1 diabetes mechanism.
\end{abstract}

Keywords Autoimmune disease $\cdot I F I H 1 \cdot$ Genetic susceptibility · Genotype · Interferon induced with helicase $\mathrm{C}$ domain $1 \cdot$ Transmission disequilibrium test .

Type 1 diabetes · Picornavirus infection .

Single-nucleotide polymorphism $\cdot$ Validation

$\begin{array}{ll}\text { Abbreviations } \\ \text { LD } & \text { linkage disequilibrium } \\ \text { OR } & \text { odds ratio } \\ \text { SNP } & \text { single-nucleotide polymorphism }\end{array}$

Electronic supplementary material The online version of this article (doi:10.1007/s00125-007-0895-6) contains supplementary material, which is available to authorised users.

H.-Q. Qu • L. Marchand · R. Grabs • C. Polychronakos $(\square)$ Endocrine Genetics Lab, The McGill University Health Center (Montreal Children's Hospital),

2300 Tupper,

Montreal, QC, Canada, H3H 1P3

e-mail: constantin.polychronakos@mcgill.ca

\section{Introduction}

Recently, a large-scale screening of 12,000 non-synonymous single-nucleotide polymorphisms (SNPs) across the human genome [1] found an association between type 1 diabetes and a non-synonymous SNP (Ala946Thr) at the IFIHI gene, with the evidence coming from both a case-control approach and a family-based approach. IFIHI is a putative RNA helicase, upregulated by interferons, especially $\beta$ interferon [2]. Ifihl-deficient mice are highly susceptible to picornavirus infection, which suggests that IFIH1 is critical for innate antivirus responses [3]. This is of particular interest given evidence for a role of viral infection in type 1 diabetes [4]. In addition to a proven causal link between type 1 diabetes and congenital rubella [5], there is evidence suggesting an association with enterovirus and picornavirus infections [6]. Although the genetic effect size found was relatively small [odds ratio $(\mathrm{OR})=0.85$ ], it suggests a potentially important role of innate immunity and interferon responses in the pathogenesis of type 1 diabetes, which may reveal therapeutic targets. The purpose of the current study was to replicate the genetic finding in an independent, family-based Canadian population sample.

\section{Methods}

Participants In this study, 589 type 1 diabetes nuclear family trios (1,767 individuals) were genotyped. Genomic DNA was obtained after informed consent. The Research Ethics Board of the Montreal Children's Hospital and other participating centres approved the study. Ethnically, participants were of mixed European descent, with the largest single group being of Quebec French-Canadian origin (40\% of total cohort). All patients were diagnosed with type 1 diabetes while younger than 18 years old and had required insulin treatment continuously from the time of diagnosis. 
Table 1 Five SNPs from the IFIH1 locus in this study

\begin{tabular}{lll}
\hline SNP & Chr2 position & Gene \\
\hline rs2111485 & $162,936,043$ & 3' flanking of $I F I H 1$ \\
rs1990760 & $162,949,558$ & nsSNP (Ala946Thr) of $I F I H 1$ \\
rs3747517 & $162,954,331$ & nsSNP (His848Arg) of $I F I H 1$ \\
rs17783344 & $163,034,400$ & nsSNP (Ala80Ser) of GCA \\
rs984971 & $163,050,028$ & 3' flanking of GCA \\
\hline
\end{tabular}

ns, non-synonymous

SNP selection Five SNPs were genotyped in our study [Table 1; Electronic supplementary material (ESM) Fig. 1]. Four of these, i.e. rs2111485, rs1990760, rs3747517 and rs984971, were found to be associated with type 1 diabetes [1], the most significant being rs1990760. The remaining three were in tight linkage disequilibrium (LD) with the condition $\left(r^{2}=0.61-0.92\right)$ [1]. The fifth SNP, rs 17783344, is flanked by the type 1 diabetes-associated SNPs in this locus ( $80 \mathrm{~kb}$ to the telomeric side of rs3747517 and $16 \mathrm{~kb}$ to the centromeric side of rs984971), but was not associated with type 1 diabetes [1]. It is a non-synonymous SNP (Ala80Ser) of the $G C A$ gene and maps to a conserved functional domain (calcium binding). We decided to include it because, according to HapMap data (http://www.hapmap. org/), rs17783344 is common in Europeans but nonpolymorphic in East Asians and Africans. This dramatic population differentiation may cause a biased estimation in a case-control study (including false negative results) because of potential population stratification. Our study on the type 1 diabetes association of rs17783344 is immune to population stratification.

Genotyping Genotypes for this study were obtained using the Sequenom iPLEX assay (Sequenom, Cambridge, MA, USA), which has been described in detail in a previous study [7]. The remaining SNPs in the panel were chosen to replicate some candidate-gene findings. One Mendelian error $(0.17 \%)$ was found in the genotyping of rs 2111485 ; two Mendelian errors $(0.34 \%)$ were found in the genotyping of rs1990760. None were found in the genotyping of the other three SNPs.

Statistics Linkage disequilibrium analyses were performed by Haploview software (http://www.broad.mit.edu/personal/ jcbarret/haploview) [8]. Genetic association was tested by the family-based association test software (http://www. biostat.harvard.edu/ fbat/fbat.htm) [9]. Using logistic regression, the OR was estimated based on the transmission disequilibrium test and the UNPHASED software package (http://www.mrc-bsu.cam.ac.uk/personal/frank/software/ unphased/) [10].

\section{Results and discussion}

The family-based association test in our study is shown in Table 2. The four SNPs reported to be associated with type 1 diabetes, i.e. rs2111485, rs1990760, rs3747517 and rs984971, showed a trend of association with type 1 diabetes in the same direction as previously reported [1]. Two SNPs, rs2111485 and rs984971, have replicable type 1 diabetes association in our dataset, with statistical significance at $\alpha=0.05$. The lack of statistical significance of the other two SNPs, rs1990760 and rs3747517, may be due to the lack of statistical power, which is $\sim 61 \%$ for rs 1990760 with $\mathrm{OR}=0.85$ at $\alpha=0.05$ level and $\sim 49 \%$ for $\mathrm{rs} 3747517$ with $\mathrm{OR}=0.86$ at $\alpha=0.05$ level. No association was found for the non-synonymous SNP rs17783344 from the GCA gene.

The type 1 diabetes-associated SNPs, rs2111485 and rs984971, locate in a LD block containing four genes: FAP, IFIH1, GCA and KCNH7 (ESM Fig. 2). The FAP gene product has been identified as a human stromal antigen, which can stimulate cytotoxic $\mathrm{T}$ cell responses [11]. The $G C A$ gene product is abundant in neutrophils and macrophages, and is associated with degranulation and conse-

Table 2 Family-based association tests of the IFIHI SNPs

\begin{tabular}{|c|c|c|c|c|c|c|c|}
\hline SNP & $\begin{array}{l}\text { Minor allele } \\
\text { (frequency) }\end{array}$ & $\begin{array}{l}\text { Hardy-Weinberg } \\
p \text { value }\end{array}$ & $\begin{array}{l}\text { Genotyping } \\
\text { success rate (\%) }\end{array}$ & $\begin{array}{l}\text { Informative } \\
\text { family number }^{\mathrm{a}}\end{array}$ & $\begin{array}{l}z \text { value (single-sided } \\
p \text { value) }\end{array}$ & $\begin{array}{l}\text { Transmitted/ } \\
\text { untransmitted }\end{array}$ & OR $(95 \% \mathrm{CI})^{\mathrm{b}}$ \\
\hline rs2111485 (A/G) & A $(0.404)$ & 0.480 & 98.0 & 355 & $-1.97(0.0244)$ & $212: 252$ & $0.84(0.70-1.00)$ \\
\hline rs1990760 (C/T) & $\mathrm{C}(0.401)$ & 0.291 & 98.1 & 346 & $-1.53(0.0630)$ & $212: 248$ & $0.87(0.73-1.04)$ \\
\hline rs3747517 (A/G) & $\mathrm{A}(0.260)$ & 0.490 & 98.2 & 319 & $-0.80(0.2119)$ & $186: 201$ & $0.92(0.76-1.12)$ \\
\hline rs17783344 (G/T) & $\mathrm{G}(0.147)$ & 0.338 & 99.9 & 224 & $-0.19(0.4247)$ & 119:122 & $0.98(0.77-1.25)$ \\
\hline rs984971 (A/G) & $\mathrm{G}(0.362)$ & 0.912 & 99.6 & 369 & $-1.69(0.0455)$ & $220: 257$ & $0.85(0.71-1.02)$ \\
\hline
\end{tabular}

\footnotetext{
${ }^{a}$ Number of nuclear families informative for (with a non-zero contribution to) family-based association test analysis

${ }^{\mathrm{b}}$ The OR of the minor allele, with the major allele as reference, was estimated by the transmission disequilibrium test using the UNPHASED software package (http://www.mrc-bsu.cam.ac.uk/personal/frank/software/unphased/)
} 
quent immune reaction [12]. Beside the antivirus-related type 1 diabetes mechanism of IFIH1, FAP and GCA may participate in the autoimmune or inflammatory destruction of pancreatic beta cells. Therefore, all of these three genes are candidates for type 1 diabetes. However, further variant discovery and fine mapping are required to confirm that the IFIH1 SNP is actually causative. Because of the tight LD and the small effect size of type 1 diabetes association, this may not be easy. However, if the antivirus-related genetic effect exists, an association between the SNPs and enterovirus infection could be found by a case-control study of host susceptibility. Moreover, if an autoimmune effect were found to exist, association between the SNPs and other cytotoxic autoimmune diseases could be expected, bearing in mind that other autoimmune diseases could also be associated with virus infection. A combined genetic effect of these genes, i.e. antivirus-associated effect and cytotoxic autoimmune reactions, is possible.

Acknowledgements This work was funded by the Juvenile Diabetes Research Foundation International and Genome Canada. H.-Q. Qu is supported by a fellowship from the Canadian Institutes of Health Research.

Duality of interest The authors declare that there is no duality of interest associated with this manuscript.

\section{References}

1. Smyth DJ, Cooper JD, Bailey R et al (2006) A genome-wide association study of nonsynonymous SNPs identifies a type 1 diabetes locus in the interferon-induced helicase (IFIH1) region. Nat Genet 38:617-619

2. Kang DC, Gopalkrishnan RV, Wu Q, Jankowsky E, Pyle AM, Fisher PB (2002) mda-5: An interferon-inducible putative RNA helicase with double-stranded RNA-dependent ATPase activity and melanoma growth-suppressive properties. Proc Natl Acad Sci U S A 99:637-642

3. Kato H, Takeuchi O, Sato S et al (2006) Differential roles of MDA5 and RIG-I helicases in the recognition of RNA viruses. Nature 441:101-105

4. van der Werf N, Kroese FG, Rozing J, Hillebrands JL (2007) Viral infections as potential triggers of type 1 diabetes. Diabetes Metab Res Rev 23:169-183

5. Menser MA, Forrest JM, Bransby RD (1978) Rubella infection and diabetes mellitus. Lancet 1:57-60

6. Haverkos HW, Battula N, Drotman DP, Rennert OM (2003) Enteroviruses and type 1 diabetes mellitus. Biomed Pharmacother 57:379-385

7. Qu H-Q, Marchand L, Grabs R, Polychronakos C (2007) The IRF5 polymorphism in type 1 diabetes. J Med Genet 44:670 672

8. Barrett JC, Fry B, Maller J, Daly MJ (2005) Haploview: analysis and visualization of LD and haplotype maps. Bioinformatics 21:263-265

9. Horvath S, Xu X, Laird NM (2001) The family based association test method: strategies for studying general genotype-phenotype associations. Eur J Hum Genet 9:301-306

10. Dudbridge F (2003) Pedigree disequilibrium tests for multilocus haplotypes. Genet Epidemiol 25:115-121

11. Fassnacht M, Lee J, Milazzo C et al (2005) Induction of CD4(+) and $\mathrm{CD} 8(+) \mathrm{T}$-cell responses to the human stromal antigen, fibroblast activation protein: implication for cancer immunotherapy. Clin Cancer Res 11:5566-5571

12. Boyhan A, Casimir CM, French JK, Teahan CG, Segal AW (1992) Molecular cloning and characterization of grancalcin, a novel EF-hand calcium-binding protein abundant in neutrophils and monocytes. J Biol Chem 267:2928-2933 